\title{
Low temperature relaxational dynamics of the Ising chain in a transverse field
}

\author{
Subir Sachdev \\ Department of Physics, Yale University \\ P.O. Box 208120, New Haven, CT 06520-8120 \\ A.P. Young \\ Department of Physics, University of California \\ Santa Cruz CA 95064
}

(September 16, 1996)

\begin{abstract}
We present asymptotically exact results for the real time order parameter correlations of a class of $d=1$ Ising models in a transverse field at low temperatures $(T)$ on both sides of the quantum critical point. The correlations are a product of a $T$-independent factor determined by quantum effects, and a $T$-dependent relaxation function which comes from a classical theory. We confirm our predictions by a no-free-parameter comparison with numerical studies on the nearest neighbor spin-1/2 model.
\end{abstract}

Real time, non-zero temperature $(T)$, correlation functions of quantum many body systems are directly related to the observables of many experiments. For strongly interacting systems, there are few quantitative results on the relaxation and transport processes that are believed to occur at long times at any $T \neq 0$. Monte Carlo and perturbative methods work best in imaginary time, but the analytic continuation to real time is most dangerous, and often fails, in the low frequency limit. At special conformally invariant points in dimension $d=1$, real time correlations describing relaxation of an order parameter can be computed exactly. Among systems in arbitrary $d$, which are tuned across a quantum critical point by a variable coupling, there is only one for which reliable results are available: the $d=1$ impenetrable Bose gas, whose correlators were determined by profound and sophisticated inverse scattering analysis 1 .

In this paper, we study real time, $T \neq 0$ correlations of the $d=1$ Ising model in a transverse field. We use a novel semiclassical method to obtain the exact asymptotics of order parameter correlations in the two low $T$ regions on either side of its quantum critical point. Our main new result, given in Eqs. (4), (8) below, is that in these low $T$ regimes, the spin correlation function can be expressed as a product of two factors. One, arising from quantum effects, gives the $T=0$ value of the correlation function, and the other, which comes from a classical theory, describes the effects of temperature. Combined with earlier results 2 3, our new results give a complete description of time-dependent correlations in all the distinct limiting regions of the Ising model, and exhibit, simply and clearly, the crossovers in the roles of quantum and thermal fluctuations in the relaxational dynamics.

For the Ising chain with only nearest neighbor exchange, we also use the free fermion representation 4 to obtain accurate, numerical data 6. for real time spin correlations for systems with up to 512 spins: these results are in excellent agreement with the asymptotic theoretical results even at relatively short times and distances.
We also use our semiclassical method to obtain correlators of the $d=1$ impenetrable Bose gas in a certain low $T$ regime; our results here are in agreement with earlier work 1 , although our approach is much simpler and more physically transparent. Our method should also apply to other $d=1$ quantum models (like the non-linear sigma or sine-Gordon) with an excitation gap.

We consider the Hamiltonian

$$
H_{I}=-\sum_{i}\left(\sum_{\ell>0} J_{\ell} \sigma_{i}^{z} \sigma_{i+\ell}^{z}+g \sigma_{i}^{x}\right)
$$

where $\sigma_{i}^{z}, \sigma_{i}^{x}$ are Pauli matrices on a chain of sites $i, J_{\ell}$ $(>0)$ are short-ranged exchange constants, and $g(>0)$ is the transverse field. The ground state of $H_{I}$ is expected to have long range order with $N_{0} \equiv\left\langle\sigma^{z}\right\rangle \neq 0$ for all $g<g_{c}$, and a gap to all excitations for $g \neq g_{c}$, , see Fig 1. Away from $g=g_{c}$, the low lying states consist

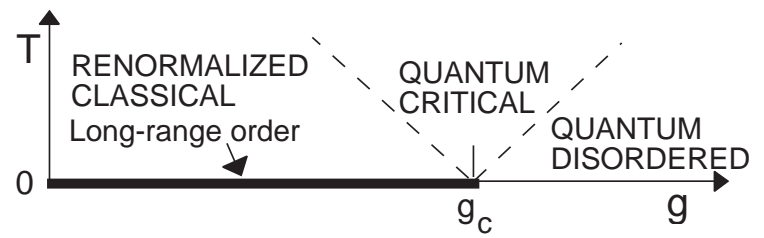

FIG. 1. Finite $T$ phase diagram of $H_{I}$. The dashed lines are crossover boundaries at $T \sim\left|m c^{2}\right|$.

of a stable particle with energy $\epsilon_{p}$ at momentum $p$, and $n>1$ particle continua at higher energy. We also have $\epsilon_{-p}=\epsilon_{p}$, and expect that $\epsilon_{p}$ has a minimum at $p=0$. Consequently, for small $p$ we parameterize $\epsilon_{p}=\left(\left(m c^{2}\right)^{2}+\right.$ $\left.c^{2} p^{2}+\mathcal{O}\left(p^{4}\right)\right)^{1 / 2}$, which defines the 'velocity' $c>0$ and the 'mass' $m$; we choose $m>0(m<0)$ for $g<g_{c}$ $\left(g>g_{c}\right)$. The critical point at $g=g_{c}$ is described by a continuum theory with dynamic exponent $z=1$, and correlation length exponent $\nu=1$; hence $c \sim$ constant for $g$ near $g_{c}$, while $m$ vanishes linearly, $|m| \sim\left|g-g_{c}\right|$, with possibly a different slope on the two sides. 
The model with $J_{\ell>1}=0$ is integrable $\Theta$, and all parameters are known exactly: in units where $\hbar=k_{B}=1$ we have $g_{c}=J_{1}, N_{0}=\left(1-\left(g / J_{1}\right)^{2}\right)^{1 / 8}$ and $\epsilon_{p}=$ $2\left(J_{1}^{2}+g^{2}-2 J_{1} g \cos (p a)\right)^{1 / 2}$, where $a$ is the lattice spacing, so $m c^{2}=2\left(J_{1}-g\right)$. However, the existence of these stable particles is not a special feature of this integrable point. Indeed, for $g \ll g_{c}$, a particle has the simple weak-coupling interpretation as the boundary between domains with opposite orientations in $\left\langle\sigma^{z}\right\rangle$. Conversely, for $g \gg g_{c}$, the ground states has all the spins oriented in the $+x$ direction, and the particle is a $-x$ spin hopping from site to site. We expect that these interpretations remain correct at the lowest energies, as we approach $g_{c}$ from either side, but not at $g=g_{c}$.

In the above interpretations, the particles are evidently bosons. They have short-range repulsive interactions, which, by an elementary calculation implies that the two-particle $S$-matrix $S_{p p^{\prime}}$ approaches -1 for $p a, p^{\prime} a \ll 1$. The integrable model has $S_{p p^{\prime}}=-1$ for all $p, p^{\prime}$, and this is often used to obtain a free-fermion description after a non-local gauge transformation: we shall not use this transformation in our analytical computations.

Our new analytical results are in the two low $T$ regimes on either side of $g_{c}$, with $T \ll\left|m c^{2}\right|$. In these regimes the density, $\rho$, of thermally excited particles $\sim e^{-\left|m c^{2}\right| / T}$, is exponentially small, their mean spacing is much larger than their thermal de Broglie wavelength, $\sim(2 m T)^{-1 / 2}$. These are the standard conditions for classical behavior in which $\rho=\int d p /(2 \pi) e^{-\epsilon_{p} / T}$.

We now derive results in the "renormalized classical" (RC) region $g<g_{c}, T \ll m c^{2}$. Consider the correlation function

$$
C\left(x_{i}, t\right)=\operatorname{Tr}\left(e^{-H_{I} / T} e^{i H_{I} t} \sigma_{i}^{z} e^{-i H_{I} t} \sigma_{0}^{z}\right) / Z,
$$

where $x_{i}=i a, Z=\operatorname{Tr} e^{-H_{I} / T}$. This can be evaluated in terms of the trajectories of the dilute gas of classical particles noting that, since the particles physically represent domain walls, $\sigma^{z}$ changes sign every time a particle goes by. Observe that the classical trajectories remain straight lines across collisions because the momenta before and after the collision are the same in $d=1$. This implies that the trajectories are simply independently distributed straight lines, placed with a uniform density $\rho$ along the $x$ axis, with an inverse slope $v_{p} \equiv d \epsilon_{p} / d p$, and with their momenta chosen with the Boltzmann probability density $e^{-\epsilon_{p} / T} / \rho$ (Fig 2). This heuristic argument, based on the classical picture, can be justified by taking the semi-classical (stationary phase) limit of the the double-time ('Keldysh') path integrall, in which each collision appears in both the forward and backward paths (generated by the $e^{-i H_{I} t}$ and the $e^{i H_{I} t}$ in (2) respectively) and therefore contributes the factor $\left|S_{p p^{\prime}}\right|^{2}=1$.

Computing $C(x, t)$ is now an exercise in classical probabilities. The value of $\sigma^{z}(0,0) \sigma^{z}(x, t)$ is the square of the magnetization renormalized by quantum fluctuations $\left(N_{0}^{2}\right)$, times $(-1)$ if the number of trajectories intersecting the dashed line in Fig. 2 is odd. Consider an Ising

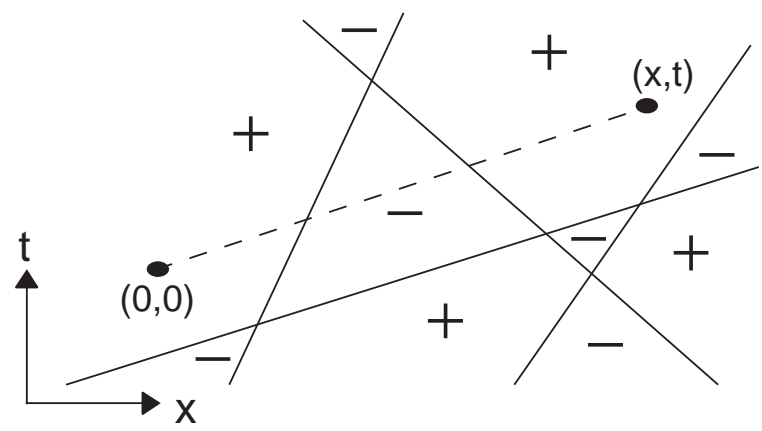

FIG. 2. A typical semiclassical contribution to the double time path integral for $C(x, t)$. Full lines are thermally excited particles which propagate forward and backward in time. The \pm signs are significant only for $g<g_{c}$ and denote the orientation of the order parameter. For $g>g_{c}$, the dashed line is a particle propagating only forward in time from $(0,0)$ to $(x, t)$.

system of size $L \gg|x|$, and let it contain $N$ thermally excited particles; then $\rho=N / L$. Let the probability that any given trajectory intersect the dashed line $=q$; then the probability only a given set of $k$ lines will intersect is $=q^{k}(1-q)^{N-k}$. Summing over all possibilities, we have

$$
\begin{aligned}
C(x, t) & =N_{0}^{2} \sum_{k=0}^{N}(-1)^{k} q^{k}(1-q)^{N-k} N ! /(k !(N-k) !) \\
& =N_{0}^{2}(1-2 q)^{N} \approx N_{0}^{2} e^{-2 q N} .
\end{aligned}
$$

The last step holds because, as we shall now compute, $q \ll 1$. First, for $t=0$, as the density of trajectories along the $t=0$ axis is uniform, $q=|x| / L$. For $t \neq 0$, consider first all trajectories with a fixed momentum $p$ : they will intersect the dashed line if their intersection with $t=0$ axis is between ordinates 0 and $x-v_{p} t$, and so $q=\left|x-v_{p} t\right| / L$. Averaging over all $p$, and inserting in (3), we get one of our main results $\theta$ :

$$
C(x, t)=N_{0}^{2} R(x, t) \quad(\mathrm{RC} \text { region }),
$$

$$
\text { where } R(x, t)=\exp \left(-\int \frac{d p}{\pi} e^{-\epsilon_{p} / T}\left|x-v_{p} t\right|\right) .
$$

Notice that $R(x, 0)=e^{-|x| / \xi}$ and $R(0, t)=e^{-|t| / \tau}$, but the general behavior is more complicated. The correlation length $\xi=1 /(2 \rho)$. Remarkably, we find from (5) that the correlation time, $\tau$, is independent of the functional form of $\epsilon_{p}$ and depends only on the gap: $\tau=(\pi / 2 T) e^{m c^{2} / T}$. In the $T \rightarrow 0$ scaling limit, in which $\xi=(\pi / 2 m T)^{1 / 2} e^{m c^{2} / T}, R(x, t)$ obeys the scaling form $R(x, t)=\phi(|x| / \xi,|t| / \tau)$, where the scaling function $\phi$ is given by

$$
\ln \phi(\bar{x}, \bar{t})=-\bar{x} \operatorname{erf}\left(\frac{\bar{x}}{\bar{t} \sqrt{\pi}}\right)-\bar{t} e^{-\bar{x}^{2} /\left(\pi \bar{t}^{2}\right)} .
$$

Simjlar classical scaling forms have been discussed earlier3, but it was incorrectly conjectured that the scat ing functions would be those of the Glauber model10; 


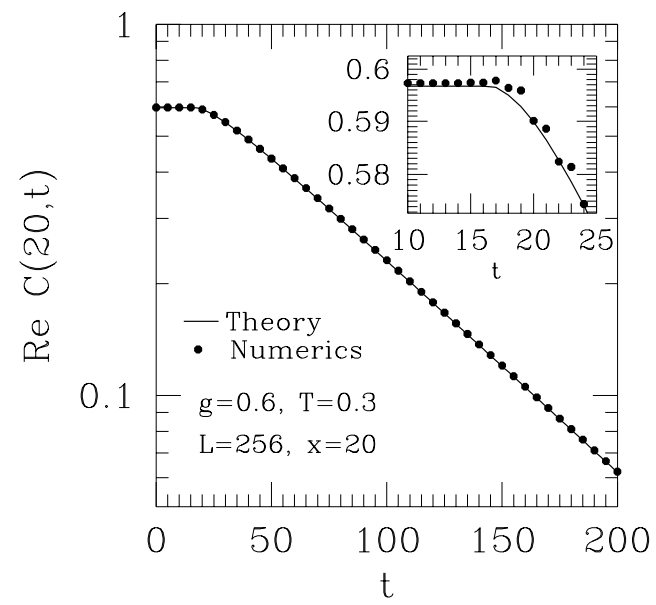

FIG. 3. The points show numerical data for the nearest neighbor model, in the $\mathrm{RC}$ region, obtained for a lattice size $L=256$ with free boundary conditions. This is compared with the theoretical prediction in Eqs. (4) and (5), in which the full lattice dispersion, $\epsilon_{p}$ was used. In the numerics, the two sites were chosen to be as close as possible to the center of the lattice.

Glauber dynamics does not conserve total energy and momentum, and these conservation laws have played a crucial role in the kinematic constraints on particle collisions.

We have also investigated the nearest neighpor model numerically by the mapping to free fermions 4 国. We generalized an earlier study 6 of equal time properties to dynamical quantitiest for the case of free boundary conditions; details will be presented elsewhere. We find that in the $\mathrm{RC}$ regime the imaginary part of $C(x, t)$ is much smaller than its real part, which suggests that the dynamics is indeed classical as argued above. In Fig. 3 we show data for $g=0.6, T=0.3$ and $x=20$ for a lattice size $L=256$. We took $J_{1}=1$ so $g_{c}=1$ and $m c^{2}=2(1-g)=0.8$. For comparison we show the theoretical prediction from Eqs. (位) and (5), in which we used the full lattice dispersion relation. The agreement is remarkably good. It is interesting to note that because there is a maximum velocity, $v_{\max }$, (on the lattice this is given by $v_{\max }=2 J_{1} g$ for $g<g_{c}$ and $v_{\max }=2 J_{1}$ for $g>g_{c}$, whereas in the continuum $v_{\max }=c$ ), Eq. (5) predicts that $C(x, t)$ should be independent of $t$ for $|t|<|x| / v_{\max }$, and the numerical results show this very clearly. The inset, with a much increased vertical scale, gives an idea of how small are the deviations between the theory and numerics. We find that the agreement is also excellent even at $r=0$, for $t>1$.

Now we turn to the "quantum disordered" (QD) region $g>g_{c}, m<0, T \ll\left|m c^{2}\right|$. The operator $\sigma^{z}$ flips spins between the $\pm x$ directions, and the large $g$ picture, noted earlier, then suggests that $\sigma^{z}$ is the sum of a creation and annihilation operator for the particles. As a result, the
$T=0$ the spectral density, obtained from the Fourier transform of $C(x, t)$, has a contribution $\sim \delta\left(\omega-\epsilon_{p}\right)$ associated with the stable particle. Higher order corrections in $1 / g$, or the form factor expansion 11 on the continuum theory valid close to $g_{c}$, show that the next contribution to the spectral density is a continuum above the 3 particle threshold. Here we will focus exclusively on how the one particle pole broadens as $T$ becomes non-zero. We define $K(x, t) \equiv C(x, t)$ at $T=0$, and dropping the multi-particle terms, we have

$$
K(x, t)=\int \frac{d p}{2 \pi} D(p) e^{i p x-i \epsilon_{p} t}
$$

where $D(p)$ is a form factor. For the general lattice model, $D(p)$ is not known; neglecting the multi-particle terms, $D(p)$ is seen from (7) to be the spatial Fourier transform of $C(x, 0)$ i.e. the structure factor. For the continuum theory, we have $D(p)=\mathcal{A} c /(2 \epsilon)$ where $\mathcal{A}$ is the dimensionless quasiparticle amplitude 11 (for an underlying integrable lattice model $\left.\mathcal{A}=2\left(\left|m c^{2}\right| / J_{1}\right)^{1 / 4}\right)$, whence $K(x, t)=\mathcal{A} K_{0}\left(m c\left(x^{2}-c^{2} t^{2}\right)^{1 / 2}\right) /(2 \pi)$, with $K_{0}$ the modified Bessel function.

Now we consider $T \neq 0$ in the semiclassical approximation. A typical set of paths contributing to the Keldysh path integral is still given by Fig 2, but its physical interpretation is now very different. The dashed line now represents the trajectory of a particle created at $(0,0)$ and annihilated at $(x, t)$, and \pm signs in the domains should be ignored. In the absence of any other particles this dashed line would contribute $K(x, t)$ to $C(x, t)$. The scattering off the background particles (the full lines in Fig 2) introduces factors of the $S$-matrix element $S_{p p^{\prime}}$; as the dashed line only propagates forward in time, the $S$-matrix elements for collisions between the dashed and full lines (and only these) are not neutralized by a complex conjugate partner. Using the low momentum value $S_{p p^{\prime}}=-1$, we see that the contribution to $C(x, t)$ equals $(-1)^{n_{\ell}} K(x, t)$ where $n_{\ell}$ is the number of full lines intersecting the dashed line. The $(-1)^{n_{\ell}}$ is precisely the term that appeared in the $\mathrm{RC}$ region, although for very different reasons. We can carry out the averaging over all trajectories as before, and obtain our main result

$$
C(x, t)=K(x, t) R(x, t) \quad(\mathrm{QD} \text { region }),
$$

where $K(x, t)$ is given by Eq. (7) and $R(x, t)$ by Eq. (5). While (7) is valid for all $x, t$, relaxation due to classical particles makes sense only within the 'light cone', and so strictly speaking, (8) requires $c t \gg x$. Outside the light cone, $K(x, t)$ decays exponentially to zero on the short length scale $\sim 1 / m c$ at which $R \approx 1$ and $T$-dependent effects are not expected to be large: so it is reasonable to use (8) except, perhaps, for $x, t$ extremely small.

The result (8) clearly displays the separation in scales at which quantum and thermal effects act. Quantum fluctuations determine the oscillatory, complex function $K(x, t)$, which gives the $T=0$ value of $C(x, t)$. Exponential relaxation of spin correlations occurs at longer 


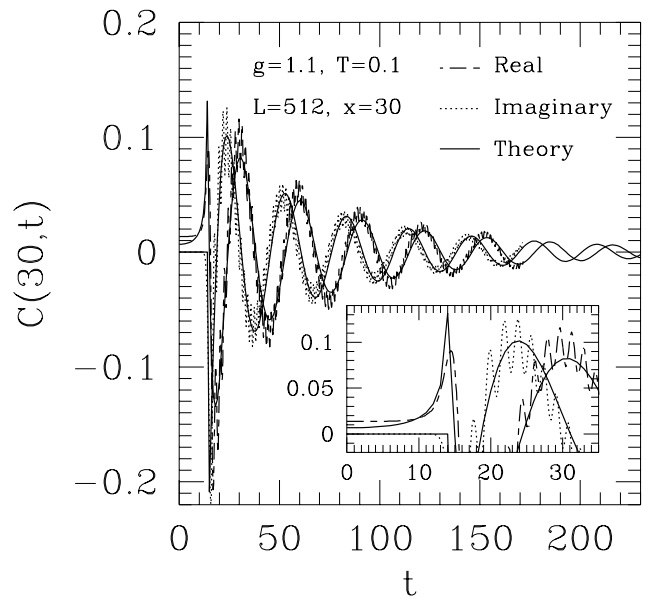

FIG. 4. The numerical data for the nearest neighbor model with $J_{1}=1$, in the QD region, obtained for a lattice size $L=512$ with free boundary conditions. Also shown is the theoretical prediction from Eqs. (8), (5) and (7), with $D(p)$ determined in the continuum theory. The numerical data has a "ringing" at high frequency, which we believe is due to the upper energy cut-off in the dispersion relation, that is present in the lattice model but not in the continuum model used for the theory. The envelope of the numerical curve fits the theoretical prediction well (but not perfectly on the scale of resolution of the figure). The insert shows a part of the same data with an enlarged scale for clarity.

scales $\sim \xi, \tau$, and is controlled by the classical motion of particles and a purely real relaxation function $R(x, t)$.

In Fig. 1 we compare the predictions of Eq. (8) with numerical results on a lattice of size $L=512$. The theoretical curve was determined from the continuum expression for $K(x, t)$, but the full lattice form for $\epsilon_{p}$ was used in Eq. (5) to determine $R(x, t)$. With the parameters used, $\left|m c^{2}\right|=0.2$, which is greater than $T(=0.1)$ as required to be in the QD region. The theory predicts that $\operatorname{Im} C(x, t)=0$ for $|t|<|x| / v_{\max }$, with a singularity in both real and imaginary parts at $|t|=|x| / v_{\max }$. At longer time both parts oscillate and decay. Part of this decay comes from the $1 / \sqrt{t}$ decay of the Bessel functions, but for $|t|>\tau$ this is dominated by the exponential decay from $R(x, t)$. The theory agrees well with the numerics; some differences are visible for small $x$, outside the light cone, but this is outside the domain of validity of (8).

Precisely the same semiclassical arguments can also be applied to the $d=1$ impenetrable gas of bosons of mass $m_{B}$, in a chemical potential $\mu<0$, with $T \ll|\mu|$. This system has $S$-matrix elements $S_{p p^{\prime}}=-1$, and its single particle propagator will be given by (8,5,17), with $\epsilon_{p}=-\mu+p^{2} / 2 m_{B}$ and $D(p)=1$. This propagator has also been computed by the inverse scattering method 1 , and the $T \ll|\mu|$ limit of their result agrees with (8).

Turning to the "quantum critical" region $\left|m c^{2}\right| \ll T \ll$ $J_{1}, g$, the dynamics is now given by finite $T$ correlators of the $g=g_{c}$ critical ppint. An explicit expression for $C(x, t)$ was given earlier $\theta_{3}$, and has exponential decay at a single time scale $\sim 1 / T$ in both its real and imaginary parts. There is no clear separation between the contributions of thermal and quantum fluctuations since both are effective at this scale. Hence there can be no effective classical model describing relaxation12. For completeness, we also note the non-universal lattice high $T$ region $T \gg J_{1}, g$; time-dependent correlators at $T=\infty$ were obtained earlier 2 and show a Gaussian decay in time.

Before concluding, we emphasize that although we compared the analytical results with numerical data on an integrable model, the main result does not depend on integrability. The simplification of the integrable model is that the parameters in the theory, $m, c, \mathcal{A}$ and $N_{0}$, are known exactly, whereas in general, they would be phenomenological parameters of a low energy theory. The equality of the classical relaxation functions in the $\mathrm{RC}$ and QD regions is surely related to the self-duality of the Ising critical theory, and is a special feature of this model. Finally, it is interesting to speculate that Eq. (8) may also be true for $d>1$ because of the separation of time scales between the quantum and thermal fluctuations.

We thank S. Majumdar, K. Damle, and T. Senthil for helpful discussions. This research was supported by NSF Grants No DMR 96-23181 and DMR 94-11964.

${ }^{1}$ Quantum Inverse Scattering Method and Correlation Functions by V.E. Korepin et. al., Cambridge University Press, Cambridge (1993), and references therein.

${ }^{2}$ H.W. Capel and J.H.H. Perk, Physica 87A, 211 (1977); J.H.H. Perk et. al. Physica 123A, 1 (1984).

${ }^{3}$ S. Sachdev, Nucl. Phys. B 464, 576 (1996); A. Leclair et. al. Nucl. Phys. B 482, 579 (1996).

${ }^{4}$ E. Lieb, T. Schultz, and D. Mattis, Ann. of Phys. 16, 406 (1961); P. Pfeuty, Ann. of Phys. 57, 79 (1970).

${ }^{5}$ B.M. McCoy et. al., Phys. Rev. A 4, 2331 (1971).

${ }^{6}$ A.P. Young and H. Rieger, Phys. Rev. B 53, 8486 (1996).

7 J. Stolze, A. Nöppert and G. Müller, Phys. Rev. B 52, 4319 (1995); H. Asakawa, Physica A 233, 39 (1996).

8 J. Rammer and H. Smith, Rev. Mod. Phys. 58, 323 (1986).

${ }^{9}$ A relaxation function similar to $R$ was also obtained in a phenomenological analysis of related models by exponentiating a short-time expansion which ignored collisions in K. Maki Phys. Rev. B 24335 (1981) and F. Devreux and J.P. Boucher J. de Physique 48, 1663 (1987); we thank O. Starykh for pointing this out to us.

${ }^{10}$ R.J. Glauber, J. Math. Phys. 4, 294 (1963).

${ }^{11}$ J.L. Cardy and G. Mussardo, Nucl. Phys. B 340, 387 (1990); V.P. Yurov and Al.B. Zamalodchikov, Int. J. Mod. Phys. A 6, 3419 (1991).

12 A.V. Chubukov et. al., Phys. Rev B 49, 11919 (1994). 